\title{
THE SHORT TIME-SCALE LIGHT VARIABILITY OF HD 153 919* REVISITED
}

\author{
E. GOS SET ${ }^{\dagger}$ \\ Institut d'Astrophysique, Université de Liège, Cointe-Ougrée, Belgium
}

(Received 5 July, 1984)

\begin{abstract}
Using Fourier analysis techniques, we have analysed all the available sets of visual photometric data concerning the X-ray binary HD 153919. This was made in order to clear up the controversy on the possible existence or absence of the small amplitude light variability $(T \sim 90 \mathrm{~min})$.

In this work, great effort has been made to keep a good level of homogeneity and objectivity. The main conclusion is that at the $99.4 \%$ confidence level, a periodicity $\left(v=15.5\right.$ day $\left.^{-1}\right)$ is detected in one data set. In this case, we can safely conclude that the short time-scale variability does exist! No other self-conclusive detection can be definitely reported; nevertheless, at the $90 \%$ confidence level, the star has a tendency to vary with characteristic frequencies of $v \sim 15 \mathrm{day}^{-1}, v \sim 30 \mathrm{day}^{-1}$ and possibly higher multiples.
\end{abstract}

\section{Introduction}

The X-ray source 4U1700 - 37 was first detected by the Uhuru satellite in December 1970 (Jones et al., 1973). The primary and confirmed optical counterpart of this X-ray binary with a period of 3.4 days is HD 153919, an O6.5f supergiant (Jones and Liller, 1973; Penny et al., 1973; Hutchings et al., 1973; Van den Heuvel, 1973; Hensberge et al., 1973; Wolff and Morrison, 1974). Optical light variations associated with the orbital motion of the system have been investigated by several authors (van Paradijs et al., 1978; hereafter referred to as PHZ; see also references given therein).

Kemp and Wolstencroft (1973) have observed light polarization in the wings of $\mathrm{H} \beta$ and remarked that, superimposed to the orbital variations, there exist other ones within a time-scale of $10 \mathrm{~min}$ or greater. Van Genderen and Uiterwaal (1976, 1978; hereafter referred to as $\mathrm{GU}$ ) have also investigated the light curve at the egress from three different primary eclipses (X-ray companion in front) and have reported variations with typical time-scale of 1-3 hr. Such fluctuations were also noticed in the X-ray domain from the Copernicus satellite (Mason et al., 1976).

Analysing SAS-3 X-ray observations, Matilsky and Jessen (1978) and Matilsky et al. (1978) claimed the possible discovery of a persistent, approximately sinusoidal, $96.8 \mathrm{~min}$ modulation. Such a long period, perhaps associated with the rotation of the X-ray companion, makes this star very interesting with respect to current theoretical models of such systems.

Due to the particular observational spectral window, mainly determined by the $94.5 \mathrm{~min}$ sidereal and the $101 \mathrm{~min}$ synodic (South Atlantic Anomaly) periods of the satellite, Hammerschlag-Hensberge et al. (1979) concluded that no certainty could be

* HD $153919 \equiv 4 \mathrm{U} 1700-37 \equiv \mathrm{V} 884$ Sco.

$\dagger$ Chercheur au 'Fonds de la Recherche Fondamentale Collective'. 
derived from those data concerning the existence or the non-existence of such an interlaced periodicity. It could be an alias of a peak corresponding to a smooth lowfrequency signal (e.g., the orbital variability).

Meanwhile, analysing the data published by PHZ, Kruszewski (1978) reported the presence of a similar periodicity on four nights corresponding to the $0.44-0.59$ binary phase range. Moreover, he tentatively identified a similar $\sim 100$ min periodicity in the GU data taken 400 days prior to PHZ. Furthermore, on the basis of new observations, Surdej (1978) has reported variations in the V Johnson filter with a $0.01 \mathrm{mag}$. amplitude and a $95 \pm 3$ min period. The variations in a narrow-band filter centered on He II $\lambda 4686$ were found to exhibit the same trend with the exception that they show a secondary minimum.

We must point out that, in their critical paper, Hammerschlag-Hensberge et al. (1979) ascribed the high pulse amplitude discovered in the PHZ data by Kruszewski (1978) to a small number of less reliable (spurious?) points. To overcome these problems, they also analysed new measurements taken in the Walraven system on the night of 29-30 May, 1978 (hereafter referred to as GW05; see Table I) and concluded to the absence of evidence of any periodicity.

TABLE I

Nomenclature of the different data sets

\begin{tabular}{lllll}
\hline $\begin{array}{l}\text { Name } \\
\text { (this work) }\end{array}$ & Date of observation & $\begin{array}{l}\text { Photometric } \\
\text { system }\end{array}$ & $\begin{array}{l}\text { Orbital } \\
\text { phase }\end{array}$ & Reference paper \\
\hline GU01 & 10 Jun., 1975 & W & 0.65 & GU \\
GU02 & 17 Jun., 1975 & W & 0.66 & GU \\
GU03 & 4 Jul., 1975 & W & 0.66 & GU \\
PHZ & 19 Aug.-22 Sep., 1976 & W & - & PHZ \\
JS01 & 15 Jun., 1978 & J & 0.52 & Kruszewski et al. (1979) \\
GW01 & 9 Jun., 1977 & W & 0.61 & GW \\
GW02 & 23 Jun., 1977 & W & 0.71 & GW \\
GW03 & 3 Jul., 1977 & W & 0.64 & GW \\
GW04 & 10 Jul., 1977 & W & 0.69 & GW \\
GW05 & 29 May, 1978 & W & 0.38 & GW \\
GW06 & 5 Jun., 1978 & W & 0.43 & GW \\
PW01 & 16 Aug., 1981 & W & 0.51 & PW \\
PW02 & 26 Aug., 1981 & W & 0.44 & PW \\
PA01 & 15 Apr., 1982 & W & 0.51 & PA \\
PA02 & 29 Apr., 1982 & W & 0.60 & PA \\
PA03 & 3 May, 1982 & W & 0.48 & PA \\
\hline
\end{tabular}

$\mathrm{J}=$ Johnson; $\mathrm{W}=$ Walraven.

In response to the letter of Hammerschlag-Hensberge et al. (1979), Kruszewski et al. (1979) made a detailed analysis of the PHZ and GU data as well as of the observations performed by Surdej (hereafter referred to as JS01; see Table I). They concluded to the existence of the periodicity and even derived a temporal variation of the period $\dot{P}$, in good 
agreement with theoretical works on disk accretion (Rappaport and Joss, 1977). Van Genderen and Windhorst (1981) and Van Genderen et al. (1981; hereafter referred to as $\mathrm{GW}$ ) reported new photometry of this star in the Walraven system. This consists of four long observing runs during 1977 and of two others during 1978. However, the 1978 data have been gathered under less favourable sky conditions than in 1977 and the authors discarded them when discussing the short time-scale (1-2 hr) variations. It is quite interesting to note that one of the two nights: namely, 29-30 May (GW05), is the one discussed by Hammerschlag-Hensberge et al. (1979). Van Genderen and Windhorst (1981) concluded from their observations that:

(a) The pulses are not always present, although the long runs are made outside the X-ray eclipse.

(b) The pulses seem to be of a shorter duration when the amplitude is high (at most 0.02 mag.).

Another set of observations has been obtained during six different nights by Van Paradijs and Van der Woerd (1982; hereafter referred to as PW). Folding their data in a phase diagram with a period of $90 \mathrm{~min}$, they attempted to show evidence for the periodicity of the variations. Essentially, this led to a negative conclusion.

Finally, looking at the time plots of additional data gathered during three different long runs in 1982, Van Paradijs and Van Amerongen (1982; hereafter referred to as PA) reported the absence of optical pulses in HD 153919.

From all these results, one can just conclude that the situation is extremely confused and even obscure: some authors claiming the discovery of well-marked (possibly periodic) variations, other ones refuting it in a rather convincing way. Furthermore, the great diversity of the methods used by the different authors in order to analyse their data makes the situation even more complex. In any case, only subjective inference has been made concerning the visual photometry.

In this paper, we attempt to clarify the situation making a homogeneous analysis of all these data. Efforts were also made to reach a comfortable level of objectivity.

\section{Analysis of the Data}

\subsection{THE DATA}

We searched the literature in order to compile all the available long runs of visual photometry of HD 153919.

These are listed in Table I. Column 1 refers to the adopted name of the data set in this work; column 2 refers to the date of the relevant observations; column 3 refers to the photometric system that was used $(\mathrm{J}=\mathrm{J}$ ohnson, $\mathrm{W}=$ Walraven $)$; column 4 refers to the relevant orbital phase, and column 5 to the reference from which the data were taken. Generally, they correspond to single nights; one exception is the original data of PHZ composed of up to 29 nights. We decided to limit ourselves to the $V$ band, since it is the only one having similarities in the two photometric systems used and, moreover, it is usually the less noisy one. 


\subsection{The TOOL OF THE ANALysis}

One of the most powerful tools for investigating the presence or absence of periodicities is the well-known Fourier analysis. Owing to the general characteristics of the data, it is advisable to use DFT (Discrete Fourier Transform) algorithm especially developped for uneven sampling such as the one of Deeming (1975). As our major goal was to reach a certain level of objectivity, it was convenient to try to give an answer to the problem in terms of the statistical theory. The theory of statistical analysis of Fourier power spectra has been made by several authors in the case of even sampling (e.g., Blackman and Tukey, 1959; Groth, 1975).

Recently, Scargle (1982) extended the problem to uneven sampling and suggested a way to estimate the SL (Significance Level)* through a modified expression of the periodogram: the PE (Periodogram Estimate).

Throughout this paper, we will use his method. The basic problem is to detect, a periodic signal hidden in random variations typically called noise. Then it is straightforward to define the null hypothesis $H_{0}$ by saying that we have to deal with data generated by a purely random process customarily called noise and, furthermore, to assume that this noise is approximately white (i.e., has the same power at all frequencies). As, on the opposite, deterministic processes (as for example periodic ones) have a tendency to exhibit a discrete power spectrum, we are going to collect the most powerful lines in the PE and to test them against the null hypothesis. However, while doing this, crucial problems can be encountered. One of these is aliasing. Due to the particular type of the sampling, the power in one unit bandwidth $P(y)$ is not only a function of the true power of the observed process at this frequency $v$ but, to some extent, also depends on the power $P\left(v^{\prime}\right)$ at a possibly distant frequency.

Let us notice that the dependence, or more rigorously the correlation, can be evaluated by estimating the observational spectral window at the frequency $\left|v-v^{\prime}\right|$. However, the statistical behaviour is more intricate. In a more general way, the problem is the power leakage. Leakage can be to nearby frequencies (e.g., sidelobes) or to more distant ones (e.g., aliasing). An unavoidable characteristic of minimizing procedures of these problems is either a statistical complication or an artificial noise amplification.

\subsection{The analysis}

Each data set has been treated separately. In order to remove the 3.4 variation, we have subtracted from the data a fitted second-degree polynomial. In each case, the PE has been investigated at least in the frequency domain $v \in[0.0,200.0]$ day $^{-1}$ and generally further. This upper limit roughly corresponds to the relevant critical frequency $v_{c}$ (the analog of the Nyquist frequency with respect to the sampling). This prevents us from discarding any zone of the power spectrum and permits to be conscious of the importance of the aliasing phenomenon. The different spectral windows teach us that this latter is

* It is convenient to define the SL as being the probability, under the null hypothesis, of observing a given value of the statistic. Clearly, if the SL is identically 0, the null hypothesis can be rejected for this observation. 
never dramatic. It is not surprising at all since it is well-known that any deviation from a pure evenness in the spacing substantially reduces the phenomenon. Moreover, the continuity of the sampling accounts for the complete lack of enforced regularities as well as of strong distant sidelobes in the spectral window.

Since we must pay the statistical penalty of inspecting a large number of frequencies, we shall calculate the significance level using Equation (14) of Scargle (1982). It is the same as saying that we have no a priori knowledge of the periodicity that we are looking for. This will naturally help preserving the objectivity of our analysis.

The next step is to gather some peaks of the PE in two sets $A$ and $B$. Set $A$ contains all the peaks having a significance level lower (i.e., 'better') than a certain limit. Conveniently, we choose for the limit $0.37(\sim 1 / e)$. Set $B$ contains the three most significant peaks of each data set. Clearly, we have $A \subset B$. This last approach has the advantage of an a posteriori minimization of the biasing effect of the power leakage.

In Section 3, we review the results. We limit ourselves to the low-frequency part of the PE. It must be clear that this does not introduce any bias as, in fact, we inspected the whole domain of available frequencies. Moreover, each time a peak was selected, we made sure that it was not predominantly the alias of a higher frequency peak.

\section{The Results}

The synopsis of the results can be found in Table II. In what follows, we are going to discuss the individual results.

\subsection{INDIVIDUAL DISCUSSIONS}

GU01 + GU02 (GU03)

Independently, the PE of GU01 and GU02 shows several low-frequency peaks. The GU03 data clearly exhibits a 'pulse' with a time-scale $\Delta t \sim 0.12$ day. The identification in the PE with the main peak at $7.8 \mathrm{day}^{-1}$ is straightforward. This last feature is strong and somewhat blended with another peak at $v \sim 14.4$ day $^{-1}$ (period $T=100 \mathrm{~min}$ ). Owing to the particular shape of the spectral window, we can be sure that the latter essentially is an alias of the former. So, as stated by Kruszewski et al. (1979), there effectively is no visible variation with $T \sim 90 \mathrm{~min}$ in GU03; however, the assumption of Kruszewski et al. (1979) that, adding GU03 to GU01 + GU02 would have the sole effect of changing the spectral window without affecting the true power spectrum near the peak at $T \sim 90 \mathrm{~min}$ is an oversimplification that is probably erroneous.

In any case, it is safe to restrict ourselves to the analysis of GU01 + GU02. The results are shown in the upper part of Table II. A first group of one-day aliases (when we analyze several nights together, one-day aliases appear, they are linked by the + symbol in Table II) at a significance level of 0.026 is quite interesting. Let us note that the envelope of this group of aliases is somewhat shouldered near $v \sim 28.0$ day $^{-1}$. The main frequency is then $v=31.7$ or 32.0 day $^{-1}$ in discordance with the results of Kruszewski et al. (1979). The third group is the one discussed by the latter authors. 
TABLE II

Position and significance level of the three highest peaks in the PE of the different data sets

\begin{tabular}{|c|c|c|c|}
\hline $\begin{array}{l}\text { Name } \\
\text { (this work) }\end{array}$ & $\begin{array}{l}\text { First peak } \\
v\left(\text { day }^{-1}\right) \\
S L\end{array}$ & $\begin{array}{l}\text { Second peak } \\
\left.v \text { (day }^{-1}\right) \\
S L^{\mathrm{a}}\end{array}$ & $\begin{array}{l}\text { Third peak } \\
v\left(\text { day }^{-1}\right) \\
S L^{\mathrm{a}}\end{array}$ \\
\hline GU01 + GU02 & $\begin{array}{l}31.7+32.0 \\
0.026\end{array}$ & $\begin{array}{l}90.0 \\
0.328\end{array}$ & $\begin{array}{l}13.6+13.9+13.3 \\
0.369\end{array}$ \\
\hline GU03 & - & - & - \\
\hline PHZ (29 nights) & $\begin{array}{l}56.8+57.8+55.8 \\
1.000\end{array}$ & $\begin{array}{l}92.8+93.8+91.8 \\
1.000\end{array}$ & $\begin{array}{l}15.4+16.4+14.4 \\
1.000\end{array}$ \\
\hline PHZ (3 nights) & $\begin{array}{l}18.6+19.6 \\
0.585\end{array}$ & $\begin{array}{l}32.9+33.9 \\
0.630\end{array}$ & $\begin{array}{l}84.6+83.6 \\
0.666\end{array}$ \\
\hline JS01 & $\begin{array}{l}15.5 \\
0.006\end{array}$ & $\begin{array}{l}29.8 \\
0.711\end{array}$ & $\begin{array}{l}39.4 \\
0.730\end{array}$ \\
\hline GW01 & - & - & - \\
\hline GW02 & $\begin{array}{l}28.2 \\
0.293\end{array}$ & $\begin{array}{l}19.9 \text { to } 23.1 \\
\sim 0.4\end{array}$ & $\begin{array}{l}14.9 \\
0.557\end{array}$ \\
\hline GW03 & - & - & - \\
\hline GW04 & $\begin{array}{l}28.8 \\
0.429\end{array}$ & $\begin{array}{l}62.1 \\
0.750\end{array}$ & $\begin{array}{l}35.0 \\
0.790\end{array}$ \\
\hline GW05 & - & - & $\begin{array}{l}57.2 \\
0.154\end{array}$ \\
\hline GW06 & $\begin{array}{l}71.9 \\
0.781\end{array}$ & $\begin{array}{l}28.9 \\
0.818\end{array}$ & $\begin{array}{l}88.8 \\
0.899\end{array}$ \\
\hline PW01 & - & - & - \\
\hline PW02 & $\begin{array}{l}11.7 \\
0.052\end{array}$ & $\begin{array}{l}63.1 \\
0.495\end{array}$ & $\begin{array}{l}36.4 \\
0.614\end{array}$ \\
\hline PA01 & - & - & $\begin{array}{l}70.1 \\
0.456\end{array}$ \\
\hline PA02 & $\begin{array}{l}6.0 \\
0.011\end{array}$ & $\begin{array}{l}54.6 \\
0.679\end{array}$ & $\begin{array}{l}14.5 \\
0.939\end{array}$ \\
\hline PA02 \# 2 & $\begin{array}{l}46.3 \\
0.354\end{array}$ & $\begin{array}{l}52.0 \\
0.606\end{array}$ & $\begin{array}{l}9.4 \\
0.762\end{array}$ \\
\hline PA03 & - & - & - \\
\hline
\end{tabular}

a The significance levels of the second and third peaks have no proper meaning; they are given only for comparison.

PHZ

The 29 nights of PHZ have been investigated as a whole. As some nightly runs are very short, the subtracted second degree polynomial can remove some short time-scale variations. So, we have also computed the $\mathrm{PE}$ after having merely removed the first trend in each night. In the two PE's, there is clearly a group of one-day aliases out of which the highest are at $v=15.4,14.4$, and 16.4 day $^{-1}$. At first glance, this could be thought to be a corroboration of the conclusion of Kruszewski et al. (1979). However, it is not. This group of peaks is far from being the highest one; moreover, the significance level is equal to 1 (i.e., in perfect accordance with the null hypothesis). This is probably due to the great number of nights during which the star does not show any variation. Clearly, no conclusion can be made from these data as they stand. Let us note that again the 
third group is the one investigated by Kruszewski et al. (1979). Upon re-examining the latter, Hammerschlag-Hensberge et al. (1979) attributed the magnitude of the pulse amplitude 'to a small number of less reliable points'. In order to improve this assumption, we deleted all the spurious points and computed again the PE: it has not significantly changed.

We have also studied a limited data set consisting of three nights for which the phase is about 0.5 . Here the PE is drastically changed. One can see three, barely outstanding, groups of peaks. The associated frequencies seem to be totally different from the usually claimed ones.

JS01

We confirm the periodogram of Kruszewski et al. (1979). It is dominated by a single peak at $v=15.5 \mathrm{day}^{-1}(T=93 \mathrm{~min})$. The significance level is good. It can still be improved if we restrict ourselves to the data after J.D. 2443675.7. In this case, the value reaches $S L=0.0008$. Both values of $S L$ are comfortable enough to assert that the periodic variation is real.

GW01

All the dominant peaks in the PE are located at high frequencies. Low-frequency peaks are totally unsignificant.

GW02

The PE is dominated by three peaks. The second one recorded in Table II is, in fact, double. No significance level is conclusive.

GW03

Only a peak at $v=64.5 \mathrm{day}^{-1}$, practically insignificant, can be noticed in the lowfrequency part of the PE.

GW04

Three peaks are clearly seen. The second one is somewhat larger than the $v=0$ peak of the spectral window.

GW05

High-frequency peaks clearly dominate the PE. Generally, it can be associated with spurious points. This tends to confirm the bad quality of the data already quoted by Van Genderen and Windhorst (1981). So, we agree with the relevant conclusion of Hammerschlag-Hensberge et al. (1979) concerning the absence of any periodicity at $T \sim 90 \mathrm{~min}$ in this data set. However, we must point out the existence of a peak at $v=57.2$ day $^{-1}$ with $S L=0.154$. We have computed the PE of several high-frequency sine waves sampled in exactly the same way as the star was. No alias appears at the relevant frequency. 


\section{GW06}

Up to five peaks are clearly present. The three highest ones are listed in Table II. They are essentially insignificant.

PW01

Again, spurious points seem to induce high-frequency peaks.

\section{PW02}

Only three peaks dominate the PE. The highest one $\left(v=11.7\right.$ day $\left.^{-1}\right)$ has an interesting $S L$ of 0.052 .

\section{PA01}

Only one peak can be noticed in the low-frequency part. It is not the highest one.

\section{PA02}

An outstanding peak at $v=6.0 \mathrm{day}^{-1}$ is quite noticeable. However, some care must be taken. In fact, this periodicity is due to the discontinuity in the light variation visible at $U T=4.5 \mathrm{hr}$. It is no more than an artefact of the orbital variation. We have tried to disentangle at best the problem by taking great care in the way we removed the latter. The PA02 \# 2 symbol refers to the resulting data set. The two peaks at $v=46.3$ and at $v=52.0 \mathrm{day}^{-1}$ are somewhat blended. The variation associated with these peaks can be easily seen in Figure 2 of Van Paradijs and Van Amerongen (1982) between $U T=7$ and $U T=9.5 \mathrm{hr}$.

$P A 03$

The PE is a forest of peaks in good agreement with the characteristics of white noise.

\subsection{General DisCUSSION}

The detection of a periodicity is only conclusively effective in one case: JS01. The other cases, although sometimes interesting, are never conclusive.

The results summarized in Table II are also shown in Figure 1a. The ordinate refers to the position in frequency of the selected peaks expressed in day $^{-1}$; the abscissae are discrete and refer to the name of the analysed data set. Vertical bars represent the full width at half maximum of the relevant peak.

It is clear that, if we have to deal with white noise, the position of the collected peaks can be approximately considered as a random variable with a uniform distribution. In this context, we have constructed Figure $1 \mathrm{~b}$ which is the histogram of the positions visible in Figure 1a. Hatched squares refer to peaks belonging to set $A$ (see Section 2.3). In both set $A$ and set $B$, there seems to be a slight tendency for the peaks to cluster around $v \sim 30 \mathrm{day}^{-1}$. Nevertheless, it is easy to understand that the eyes can be misled. It is a well-known fact that the human eye is excellent at picking out unusual features, sometimes unreal. These can be induced by the particular way the binning was made. 


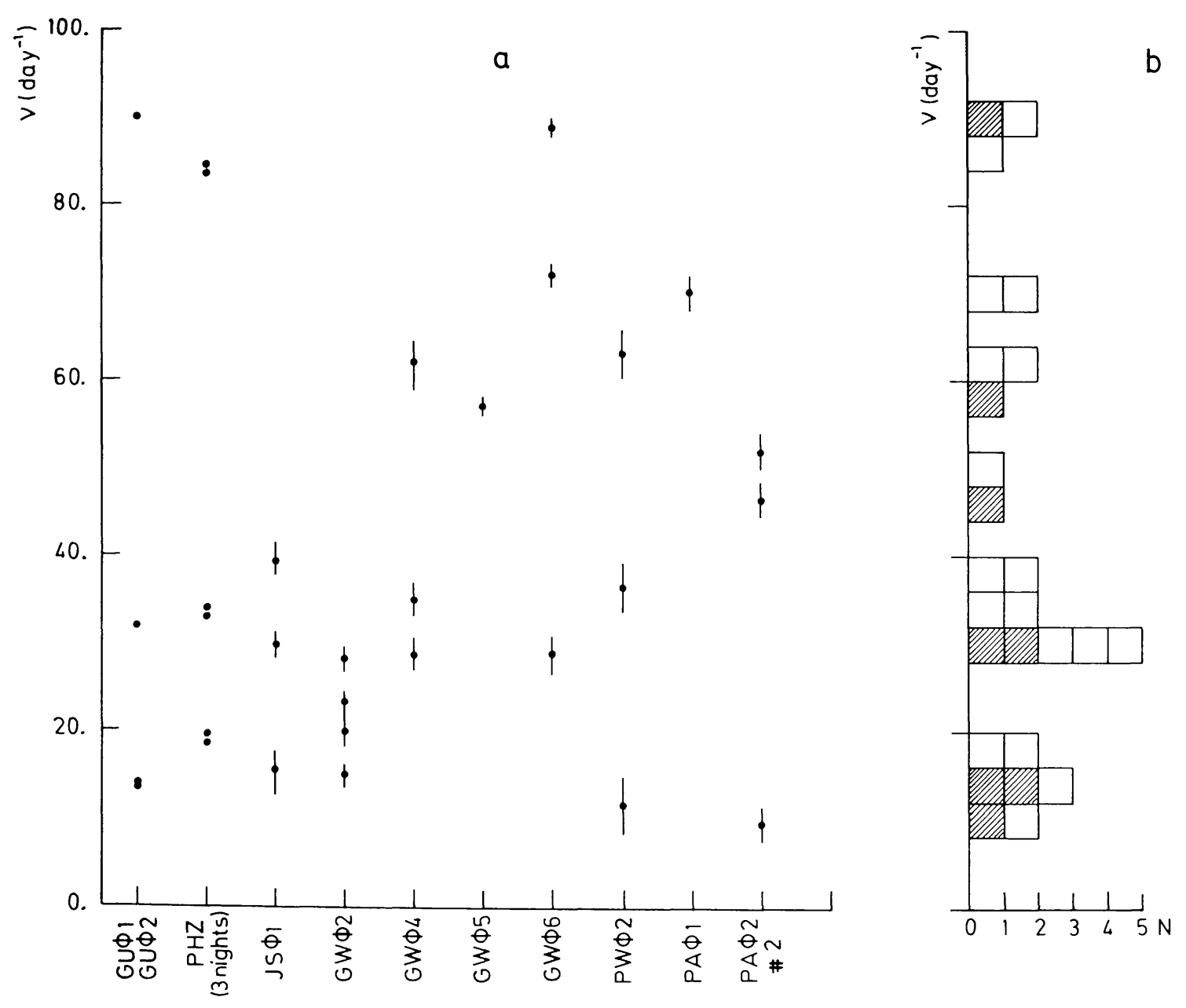

Fig. 1. (a) Positions of the different selected peaks. The ordinate refers to the frequency expressed in day $^{-1}$. The abscissae are discrete and refer to the name of the analysed data set. Vertical bars represent the full width at half maximum of the relevant peak. (b) Histogram of the positions shown in part a. The ordinate is the same. The abscissae give the number of peaks. Hatched squares represent peaks of set $A$.

Furthermore, there is always some information lost in binning. To keep aloof from such a subjectivity, it is convenient to analyse the distribution of the positions with essentially continuous statistical tests. We have chosen two of them: the first is the well-known Kolmogorov-Smirnov test; while the second one is the one-dimensional PSA (Power Spectrum Analysis), as described by Webster (1976). We now define the null hypothesis by admitting that the position is a random variable having a uniform distribution.

The two-sided Kolmogorov-Smirnov test discloses us that the main deviation from the uniform distribution occurs near $v \sim 30$ day $^{-1}$. However, the significance level is uninteresting for set $A$ whereas it is of $\sim 0.15$ for set $B$. This is barely remarkable. So we do not expect the PSA to tell us more. Figure $2 \mathrm{a}, \mathrm{b}, \mathrm{c}$ represent the run of the $Q^{\prime}$ 

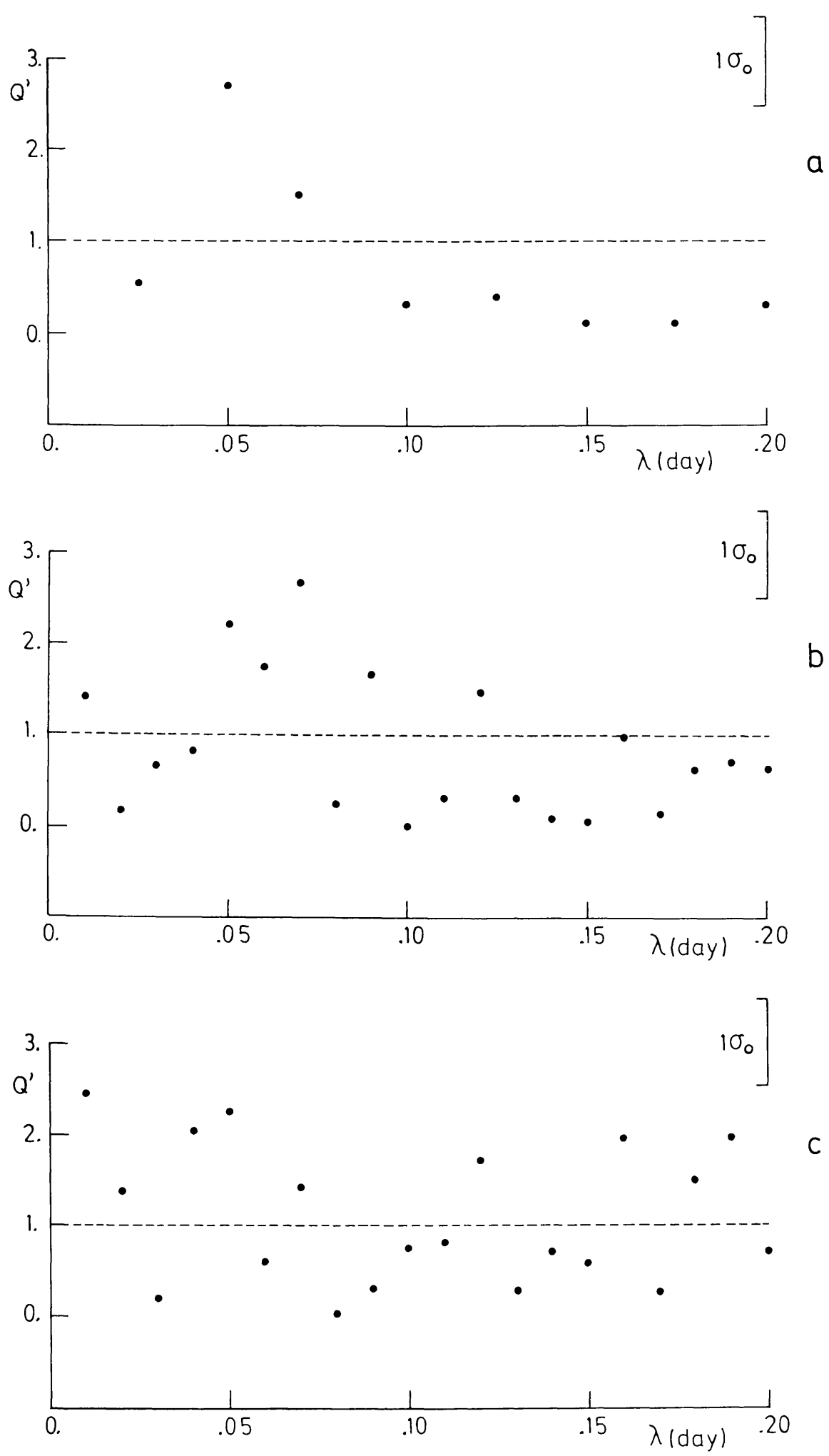

Fig. 2. Runs of the PSA $Q^{\prime}$ statistics against time-wavelength for different sets of peaks: (a) set $A$ between 0 and 40 day $^{-1}$; (b) set $A$ between 0 and 100 day $^{-1}$; (c) set $B$ between 0 and 100 day $^{-1}$. The ordinates refer to $Q^{\prime}$. Abscissae refer to the time-wavelength expressed in day. Error bars represent one standard deviation under the null hypothesis. 
statistic (see Webster, 1976) against the time-wavelength* for set $A$ between 0 and 40 day $^{-1}$, set $A$ between 0 and 100 day $^{-1}$ and, finally, set $B$ between 0 and 100 day $^{-1}$, respectively. The amazing fact is that values of the $Q^{\prime}$ statistics such as 2 do appear. In fact, in the area between $\lambda \sim 0.05$ and $\lambda \sim 0.07$ day, the $Q^{\prime}$ statistic reaches its highest level in all three graphs. The significance levels associated with such observations are $S L_{a}=0.066, S L_{b}=0.068$, and $S L_{c}=0.100$, respectively: this is not conclusive, yet quite remarkable. A detailed analysis of Figure $1 \mathrm{~b}$ helps us to explain this. The first group of positions is clustered around $v \sim 15$ day $^{-1}$, the second and most significant one around $v \sim 30 \mathrm{day}^{-1}$, and perhaps a third one around $v \sim 45 \mathrm{day}^{-1}$. Now, PSA is the very test to detect a periodic clustering (regular distribution) of this sort; on the contrary, the Kolmogorov-Smirnov test investigates a distribution through its deviation from the assumed cumulative distribution function. It will detect the maximum discordance (in this case, the one near $v \sim 30$ day $^{-1}$ ) but will discard the other ones even if they are periodic and perfectly coherent**.

At the significance level of $\sim 0.100$, the short time-scale variations of HD 153919 show a tendency to exhibit a discrete, regular, grooved power spectrum. The two main frequencies are in decreasing order of importance $v \sim 30$ day $^{-1}$ and $v \sim 15$ day $^{-1}$. This could be thought as being unphysical; perhaps it is not. Let us imagine a noiseless light curve of period $T_{0}$ with two equidistant minima differing in depth. Generally, the relevant power spectrum will have a peak at $v_{T_{0}}$ and, perhaps, a second one, distinctly smaller, at $v_{T_{1}}=2 v_{T_{0}}$. Now, let us add some noise to this hypothetical lightcurve. If the magnitude of the noise is of the order of the amplitude of the light variation, the power spectrum will hardly discern the true frequency. Even if the run is long enough to disentangle the periodic fluctuation from the noise, the power spectrum will exhibit interchangeably the frequency $v_{T_{0}}$ or the other $v_{T_{1}}$ as the most powerful peak depending on the particular behaviour (the realisation) of the noise during the observations. Moreover, the physical model of HD 153919 is not simple: several features such as a wind from the $\mathrm{O} 6.5 \mathrm{f}$ supergiant, a trailing wake behind the secondary (Hutchings, 1974a, b; Van Genderen, 1977) or an accretion disk around the relativistic companion (Balog et al., 1983) could perhaps under ad hoc hypotheses explain such an observation. In any case, such a tentative explanatory model is beyond the scope of this paper.

\section{Concluding Summary}

The paper intended to clear up the controversy on the possible existence of the photometric short-time scale variability of the X-ray binary HD 153919. Using Fourier analysis techniques, we analysed all the available long observing runs of visual photometry of this star. In order to gain in homogeneity, all data sets have been treated in

\footnotetext{
* One must point out that, in our particular case, we are investigating a frequency domain. Thus, what is generally called a frequency in PSA techniques appears here as a frequency over a frequency domain, i.e., a time-wavelength.

** Here, coherence is taken in the sense of phase coherence as in the case of the light coherence in stimulated emissions.
} 
the same way within the same method. In order to reach a good level of objectivity, data have been investigated without any a priori; furthermore, all the conclusions were formulated in terms of the statistical theory by means of the notion of 'significance level'.

Let us summarize the main results:

(1) In one data set - namely JS01 - we detect a periodicity with $v=15.5$ day $^{-1}$ $(T=93 \mathrm{~min})$ at a significance level (respectively confidence level) ranging from 0.006 (respectively $99.4 \%$ ) to 0.0008 (respectively $99.92 \%$ ). This is sufficient to conclude that it exists, in good agreement with the conclusion of Kruszewski et al. (1979).

(2) In other data sets, we can sometimes detect well marked pseudo-periodicities but the significance level is never conclusive although occasionally high. An example is GU01 + GU02 with $v=31.7$ or 32.0 day $^{-1}(T \sim 45 \mathrm{~min})$ at a significance level of 0.026 (i.e., a confidence level of $97.4 \%$ ).

(3) At a significance level of 0.100 (i.e., a confidence level of $90 \%$ ), the star variations show a tendency to have a discrete power spectrum with characteristic frequencies $v \sim 15$ day $^{-1}, v \sim 30$ day $^{-1}$, and possibly upper multiples.

(4) At a significance level of 0.15 (i.e., a confidence level of $85 \%$ ), the most commonly observed characteristic frequency is $v \sim 30$ day $^{-1}$.

Some of the results given above disagree with previous works. Statistically computed evidences are nevertheless given for the definite existence of well-defined characteristic time-scales of the system. It is clear that additional observations are necessary to better refine our conclusions.

\section{Acknowledgements}

The author is greatly indebted to J. D. Scargle for kindly providing interesting information; he further thanks J. Surdej and J. P. Swings for fruitful discussions and for reading the manuscript, as well as the Belgian 'Fonds de la Recherche Fondamentale Collective' for financial support under contracts Nos. 2.9002.76 and 2.9001.84.

\section{References}

Balog, N. I., Goncharskii, A. V., Khruzina, T. S., and Cherepashchuk, A. M.: 1983, Soviet Astron. $27,310$. Blackman, R. B. and Tukey, J. W.: 1959, The Measurement of Power Spectra, Dover Publ., New York. Deeming, T. J.: 1975, Astrophys. Space Sci. 36, 137.

Groth, E. J.: 1975, Astrophys. J. Suppl. 29, 285.

Hammerschlag-Hensberge, G., Henrichs, H. F., and Shaham, J.: 1979, Astrophys. J. 228, L75.

Hensberge, G., Van den Heuvel, E. P. J., and Paes de Barros, M. H.: 1973, Astron. Astrophys. $29,69$.

Hutchings, J. B.: 1974a, Astrophys. J. 188, 341.

Hutchings, J. B.: 1974b, Astrophys. J. 192, 677.

Hutchings, J. B., Thackeray, A. D., Webstèr, B. L., and Andrews, P. J.: 1973, Monthly Notices Roy. Astron. Soc. 163, 13P.,

Jones, C. and Liller, W.: 1973, Astrophys. J. 184, L65.

Jones, C., Forman, W., Tananbaum, H., Schreier, E., Gursky, H., Kellogg, E., and Giacconi, R.: 1973, Astrophys. J. 181, L43.

Kemp, J. C. and Wolstencroft, R. D.: 1973, Astrophys. J. 185, L21.

Kruszewski, A.: 1978, Inf. Bull. Var. Stars, No. 1424.

Kruszewski, A., Surdej, J., Zalewski, J., and Semeniuk, I.: 1979, Acta Astron. 29, 481.

Mason, K. O., Branduardi, G., and Sanford, P.: 1976, Astrophys. J. 203, L29. 
Matilsky, T. and Jessen, J.: 1978, IAU Circ., No. 3193.

Matilsky, T., La Sala, J., and Jessen, J.: 1978, Astrophys. J. 224, L119.

Penny, A. J., Olowin, R. P., Penfold, J. E., and Warren, P. R.: 1973, Monthly Notices Roy. Astron. Soc. 163, $7 \mathrm{P}$.

Rappaport, S. and Joss, P. C.: 1977, Nature 266, 683.

Scargle, J. D.: 1982, Astrophys. J. 263, 835.

Surdej, J.: 1978, IAU Circ., No. 3290.

Van den Heuvel, E. P. J.: 1973, IAU Circ., No. 2526.

Van Genderen, A. M.: 1977, Astron. Astrophys. 54, 683.

Van Genderen, A. M. and Uiterwaal, G. M.: 1976, Astron. Astrophys. 52, 139.

Van Genderen, A. M. and Uiterwaal, G. M.: 1978, Astron. Astrophys. Suppl. 34, 439; also GU.

Van Genderen, A. M. and Windhorst, R. A.: 1981, Astron. Astrophys. 97, 79.

Van Genderen, A. M., Windhorst, R. A., Van Driel, W., Bakker, R., Wesselink, T. J. H., and HammerschlagHensberge, G.: 1981, Astron. Astrophys. Suppl. 44, 83; also GW.

Van Paradijs, J. and Van Amerongen, S.: 1982, Inf. Bull. Var. Stars, No. 2180; also PA.

Van Paradijs, J., Hammerschlag-Hensberge, G., and Zuiderwijk, E. J.: 1978, Astron. Astrophys. Suppl. 31, 189; also PHZ.

Van Paradijs, J. and Van der Woerd, H.: 1982, Astron. Astrophys. 113, 27; also PW.

Webster, A.: 1976, Monthly Notices Roy. Astron. Soc. 175, 61.

Wolff, S. C. and Morrison, N. D.: 1974, Astrophys. J. 187, 69. 